\title{
A systematic review of artificial intelligence chatbots for promoting physical activity, healthy diet, and weight loss
}

Yoo Jung $\mathrm{Oh}^{1 *} \mathbb{( \mathbb { D }}$, Jingwen Zhang ${ }^{1,2}$, Min-Lin Fang $^{3}$ and Yoshimi Fukuoka ${ }^{4}$

\begin{abstract}
Background: This systematic review aimed to evaluate Al chatbot characteristics, functions, and core conversational capacities and investigate whether Al chatbot interventions were effective in changing physical activity, healthy eating, weight management behaviors, and other related health outcomes.

Methods: In collaboration with a medical librarian, six electronic bibliographic databases (PubMed, EMBASE, ACM Digital Library, Web of Science, PsycINFO, and IEEE) were searched to identify relevant studies. Only randomized controlled trials or quasi-experimental studies were included. Studies were screened by two independent reviewers, and any discrepancy was resolved by a third reviewer. The National Institutes of Health quality assessment tools were used to assess risk of bias in individual studies. We applied the Al Chatbot Behavior Change Model to characterize components of chatbot interventions, including chatbot characteristics, persuasive and relational capacity, and evaluation of outcomes.
\end{abstract}

Results: The database search retrieved 1692 citations, and 9 studies met the inclusion criteria. Of the 9 studies, 4 were randomized controlled trials and 5 were quasi-experimental studies. Five out of the seven studies suggest chatbot interventions are promising strategies in increasing physical activity. In contrast, the number of studies focusing on changing diet and weight status was limited. Outcome assessments, however, were reported inconsistently across the studies. Eighty-nine and thirty-three percent of the studies specified a name and gender (i.e., woman) of the chatbot, respectively. Over half (56\%) of the studies used a constrained chatbot (i.e., rule-based), while the remaining studies used unconstrained chatbots that resemble human-to-human communication.

Conclusion: Chatbots may improve physical activity, but we were not able to make definitive conclusions regarding the efficacy of chatbot interventions on physical activity, diet, and weight management/loss. Application of Al chatbots is an emerging field of research in lifestyle modification programs and is expected to grow exponentially. Thus, standardization of designing and reporting chatbot interventions is warranted in the near future.

Systematic review registration: International Prospective Register of Systematic Reviews (PROSPERO): CRD4202021 6761.

Keywords: Artificial intelligence, Chatbot, Conversational agent, Physical activity, Weight loss, Weight maintenance, Diet, Nutrition, Sedentary behavior, Systematic review

*Correspondence: yjeoh@ucdavis.edu

1 Department of Communication, University of California Davis, Davis, USA

Full list of author information is available at the end of the article

\section{Background}

Artificial Intelligence (AI) chatbots, also called conversational agents, employ dialogue systems to enable natural language conversations with users by means of speech, original author(s) and the source, provide a link to the Creative Commons licence, and indicate if changes were made. The images or other third party material in this article are included in the article's Creative Commons licence, unless indicated otherwise in a credit line to the material. If material is not included in the article's Creative Commons licence and your intended use is not permitted by statutory regulation or exceeds the permitted use, you will need to obtain permission directly from the copyright holder. To view a copy of this licence, visit http://creativecommons.org/licenses/by/4.0/. The Creative Commons Public Domain Dedication waiver (http://creativeco mmons.org/publicdomain/zero/1.0/) applies to the data made available in this article, unless otherwise stated in a credit line to the data. 
text, or both [1]. Powered by natural language processing and cloud computing infrastructures, AI chatbots can participate in a broad range, from constrained (i.e., rule-based) to unconstrained conversations (i.e., humanto-human-like communication) [1]. According to a Pew Research Center survey, $46 \%$ of American adults interact with voice-based chatbots (e.g., Apple's Siri and Amazon's Alexa) on smartphones and other devices [2]. The use of AI chatbots in business and finance is rapidly increasing; however, their use in lifestyle modification and health promotion programs remains limited.

Physical inactivity, poor diet, and obesity are global health issues [3]. They are well-known modifiable risk factors for cardiovascular diseases, type 2 diabetes, certain types of cancers, cognitive decline, and premature death [3-6]. However, despite years of attempts to raise awareness about the importance of physical activity (PA) and healthy eating, individuals often do not get enough PA nor do they have healthy eating habits $[7,8]$, resulting in an increasing prevalence of obesity $[9,10]$. With emerging digital technologies, there has been an increasing number of programs aimed at promoting PA, healthy eating, and/or weight loss, that utilize the internet, social media, and mobile devices in diverse populations [11-14]. Several systematic reviews and meta-analyses [15-19] have shown that these digital technology-based programs resulted in increased PA and reduced body weight, at least for a short duration. While digital technologies may not address environmental factors that constrain an individual's health environment, technology-based programs can provide instrumental help in finding healthier alternatives or facilitating the creation of supportive social groups [13, 14]. Moreover, these interventions do not require traditional in-site visits, and thus, help reduce participants' time and financial costs [16]. Albeit such potentials, current research programs are still constrained in their capacity to personalize the intervention, deliver tailored content, or adjust the frequency and timing of the intervention based on individual needs in real time.

These limitations can be overcome by utilizing AI chatbots, which have great potential to increase the accessibility and efficacy of personalized lifestyle modification programs [20,21]. Enabling AI chatbots to communicate with individuals via web or mobile applications can make these personalized programs available 24/7 [21, 22]. Furthermore, AI chatbots provide new communication modalities for individuals to receive, comprehend, and utilize information, suggestions, and assistance on a personal level [20,22], which can help overcome one's lack of self-efficacy or social support [20]. AI chatbots have been utilized in a variety of health care domains such as medical consultations, disease diagnoses, mental health support $[1,23]$, and more recently, risk communications for the COVID-19 pandemic [24]. Results from a few systematic reviews and meta-analyses suggest that chatbots have a high potential for healthcare and psychiatric use, such as promoting antipsychotic medication adherence as well as reducing stress, anxiety, and/or depression symptoms [1, 25, 26]. However, to the best of our knowledge, none of these studies have focused on the efficacy of AI chatbot-based lifestyle modification programs and the evaluation of chatbot designs and technologies.

Therefore, this systematic review aimed to describe AI chatbot characteristics, functions (e.g., the chatbot's persuasive and relational strategies), and core conversational capacities, and investigate whether AI chatbot interventions were effective in changing PA, diet, weight management behaviors, and other related health outcomes. We applied the AI Chatbot Behavior Change Model [22], designed to inform the conceptualization, design, and evaluation of chatbots, to guide our review. The systematic review provides new insights about the strengths and limitations in current AI chatbot-based lifestyle modifcation programs and can assist researchers and clinicians in building scalable and personalized systems for diverse populations.

\section{Methods}

The protocol of this systematic review was registered at the International Prospective Register of Systematic Reviews (PROSPERO) (ID: CRD42020216761). The systematic review was conducted in accordance with the Preferred Reporting Items for Systematic Reviews and Meta-analysis guidelines.

\section{Eligibility criteria}

Table 1 shows the summary of the inclusion and exclusion criteria of the study characteristics based on the PICOS framework (i.e., populations/participants, interventions and comparators, outcome(s) of interest, and study designs/type) [27]. We included peer-reviewed papers or conference proceedings that were available in full-text written in English. Review papers, protocols, editorials, opinion pieces, and dissertations were excluded.

\section{Information sources and search strategy}

In consultation with a medical librarian (MF), preplanned systematic search strategies were used for six electronic databases (PubMed, EMBASE, ACM Digital Library, Web of Science Core Collection, PsycINFO, and IEEE). A combination of MeSH/Emtree terms and keyword searches were used to identify studies on AI chatbot use in lifestyle changes; the comprehensive search strategies for each database are provided in Additional file 1 . 
Table 1 Summary of inclusion and exclusion criteria

\begin{tabular}{|c|c|c|c|}
\hline & & Inclusion criteria & Exclusion criteria \\
\hline $\mathbf{P}$ & Populations/participants & $\begin{array}{l}\text { Adults and/or children who use Al chatbots for PA, diet, } \\
\text { and/or weight management }\end{array}$ & None \\
\hline I & Interventions & $\begin{array}{l}\text { Constrained }^{\mathrm{a}} \text { and/or unconstrained } \mathrm{b}^{\mathrm{b}} \text { text and/or speech- } \\
\text { based Al chatbots operating as standalone software or } \\
\text { via a web browser or mobile application }\end{array}$ & $\begin{array}{l}\text { Chatbots that are part of virtual reality, augmented reality, } \\
\text { embodied agents, and/or therapeutic robots }\end{array}$ \\
\hline C & Comparators & $\begin{array}{l}\text { With or without a usual care group }{ }^{c} \text {, comparison group, } \\
\text { or an attention control group }\end{array}$ & None \\
\hline 0 & Outcome(s) & $\begin{array}{l}\text { Main outcomes: Changes in self-reported and/or objec- } \\
\text { tively measured PA, sedentary behavior, diet, and/or body } \\
\text { weight } \\
\text { Secondary outcomes: Feasibility, acceptability, safety } \\
\text { (e.g., adverse events, injury), and/or user satisfaction of } \\
\text { chatbots if available }\end{array}$ & $\begin{array}{l}\text { Studies that report only chatbot infrastructure or algorithm } \\
\text { designs }\end{array}$ \\
\hline$S$ & Study designs/types & $\begin{array}{l}\text { Randomized controlled trials or quasi-experimental } \\
\text { studies }\end{array}$ & $\begin{array}{l}\text { Qualitative studies, case-control studies, cross-sectional } \\
\text { studies, or cohort studies }\end{array}$ \\
\hline
\end{tabular}

Further, hand-searching was done to ensure that relevant articles were not missed during the data collection. The searches were completed on November 14, 2020. No date limits were applied to the searches.

\section{Study selection}

All retrieved references were imported into the Endnote reference management software [28], and duplicates were removed. The remaining references were imported into the Covidence systematic review software [29], and additional duplicates were removed. Before screening the articles, three researchers (YO, JZ, and YF) met to discuss the procedure for title and abstract screening using 20 randomly selected papers. In the first phase of screening, two researchers ( $\mathrm{YO}$ and JZ) independently assessed all study titles and abstracts against the eligibility criteria in Table 1. The agreement in the abstract and title screening between the two reviewers was $97.4 \%$ (Cohen's Kappa $=.725)$. Then, they (YO and JZ) read the remaining studies in full length. The agreement for full text screening was 91.9\% (Cohen's Kappa $=.734)$. Discrepancies at each stage were resolved through discussion with a third researcher (YF).

\section{Data collection process and data items}

Data extraction forms were developed based on the AI Chatbot Behavior Change Model [22], which provides a comprehensive framework for analyzing and evaluating chatbot designs and technologies. This model consists of four major components that provide guidelines to develop and evaluate AI chatbots for health behavior changes: 1) designing chatbot characteristics and understanding user background, 2) building relational capacity, 3) building persuasive capacity, and 4) evaluating mechanisms and outcomes. Based on the model, the data extraction forms were initially drafted by YF and discussed among the research team members. One researcher (YO) extracted information on study and sample characteristics, chatbot characteristics, intervention characteristics, outcome measures and results for main outcomes (i.e., PA, diet, and weight loss) and secondary outcomes (i.e., engagement, acceptability/satisfaction, adverse events, and others). Study and sample characteristics consisted of study aim, study design, theoretical framework, sample size, age, sex/gender, race/ethnicity, education, and income. Chatbot characteristics included the systematic features the chatbots were designed with (i.e., chatbot name and gender, media, user input, conversation initiation, relational capacity, persuasion capacity, safety, and ethics discussion). Intervention characteristics included information such as intervention duration and frequency, intervention components, and technological features (e.g., system infrastructure, platform). Two researchers (YF and JZ) independently validated the extracted data.

\section{Quality assessment and risk of bias}

Two reviewers (YO and JZ) independently evaluated the risk of bias of included studies using the two National Institutes of Health (NIH) quality assessment tools [30]. Randomized controlled trials (RCTs) were assessed for methodological quality using the NIH Quality Assessment of Controlled Intervention Studies. For quasiexperimental studies, the NIH Quality Assessment Tool 
for Before-After (Pre-Post) Studies with No Control Group was used. Using these tools, the quality of each study was categorized into three groups ("good," "fair," and "poor"). These tools were used to assess confidence in the evaluations and conclusions of this systematic review. We did not use these tools to exclude the findings of poor quality studies. It should be noted that the studies included in this systematic review were behavioral intervention trials targeting individual-level outcomes. Therefore, criteria asking 1) whether participants did not know which treatment group they were assigned to and 2) the statistical analyses of group-level data were considered inapplicable.

\section{Synthesis of results}

Due to the heterogeneity in the types of study outcomes, outcome measures, and clinical trial designs, we qualitatively evaluated and synthesized the results of the studies. We did not conduct a meta-analysis and did not assess publication bias.

\section{Results}

\section{Study selection}

Figure 1 shows the study selection process. The search yielded 2360 references in total, from which 668 duplicates were removed. A total of 1692 abstracts were then screened, among which 1630 were judged ineligible, leaving 62 papers to be read in full text. In total, 9 papers met the eligibility criteria and were included.

\section{Summary of study designs and sample characteristics}

The 9 included papers had been recently published (3 were published in 2020 [20,31, 32], 4 in 2019 [21, 33$35]$, and 2 in 2018 [36, 37]). Table 2 provides details of the characteristics of each study. Two studies [21, 37] were conducted in the United States and the remaining 7 were conducted in Switzerland [31,33,36], Australia [20], South Korea [32], and Italy [34] (1 not reported [35]). In total, 891 participants were represented in the 9 studies, with sample sizes ranging from 19 to 274 participants. The mean age of the samples ranged from 15.2

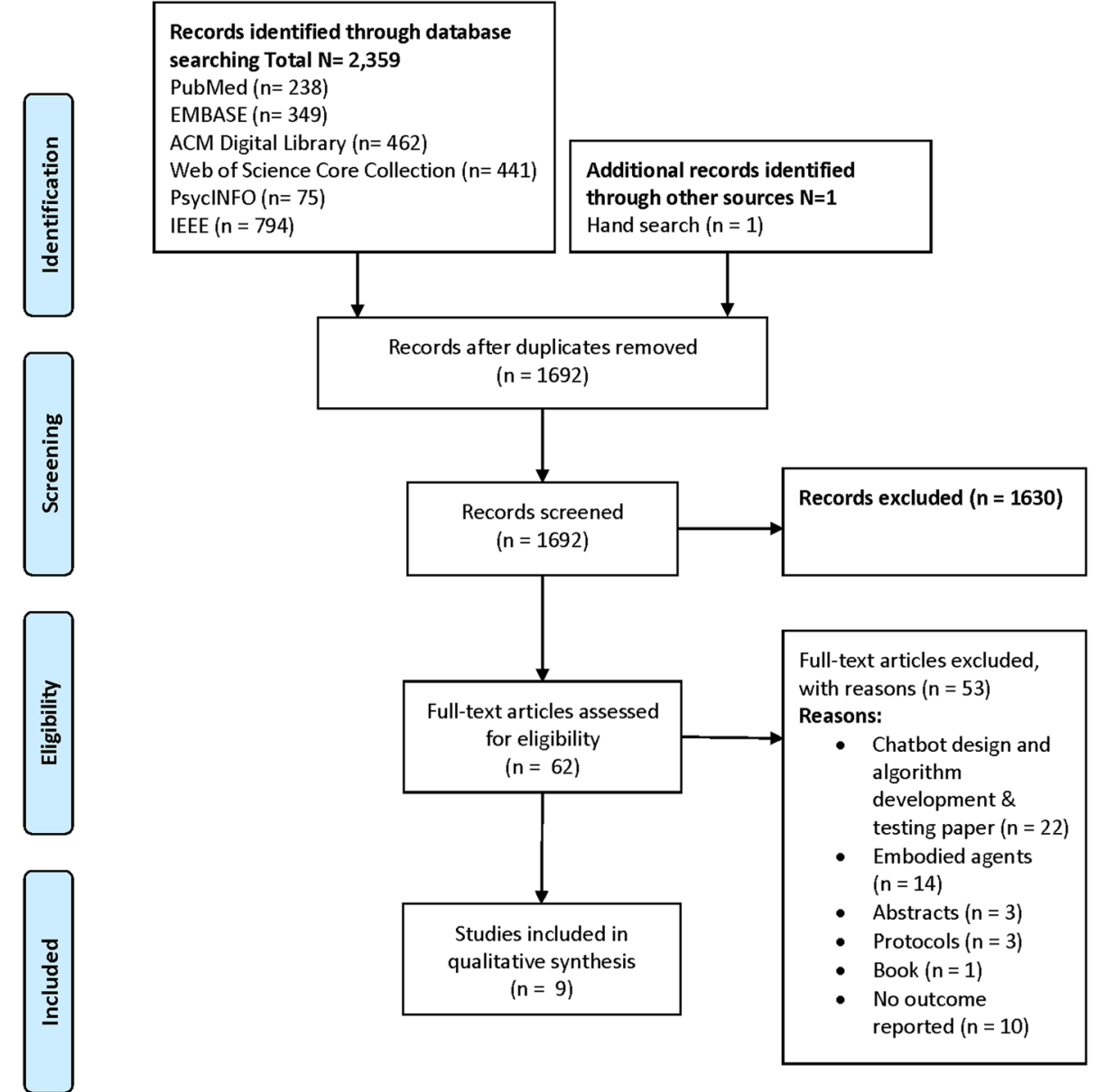

Fig. 1 Flow diagram of the article screening process 


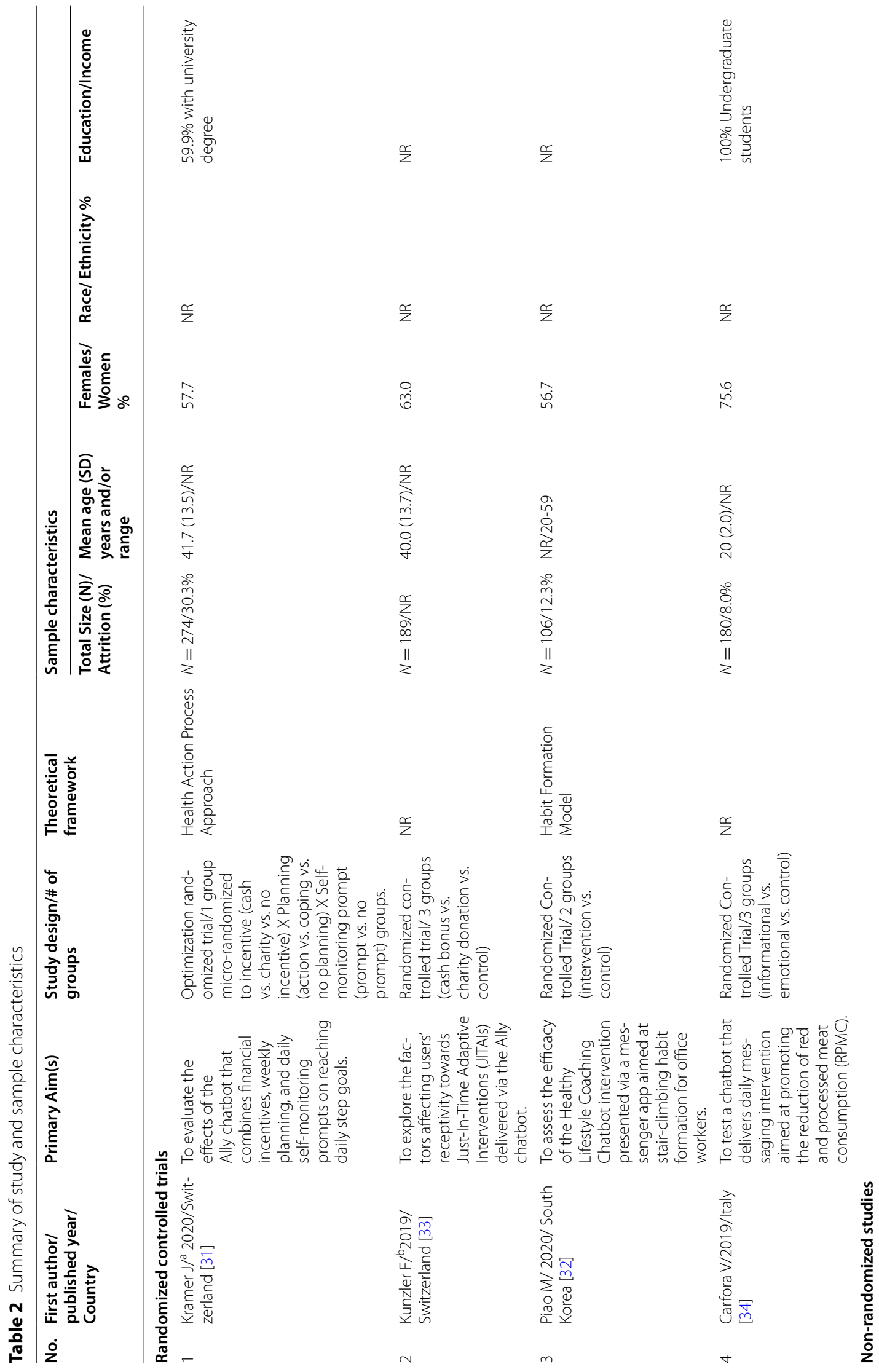




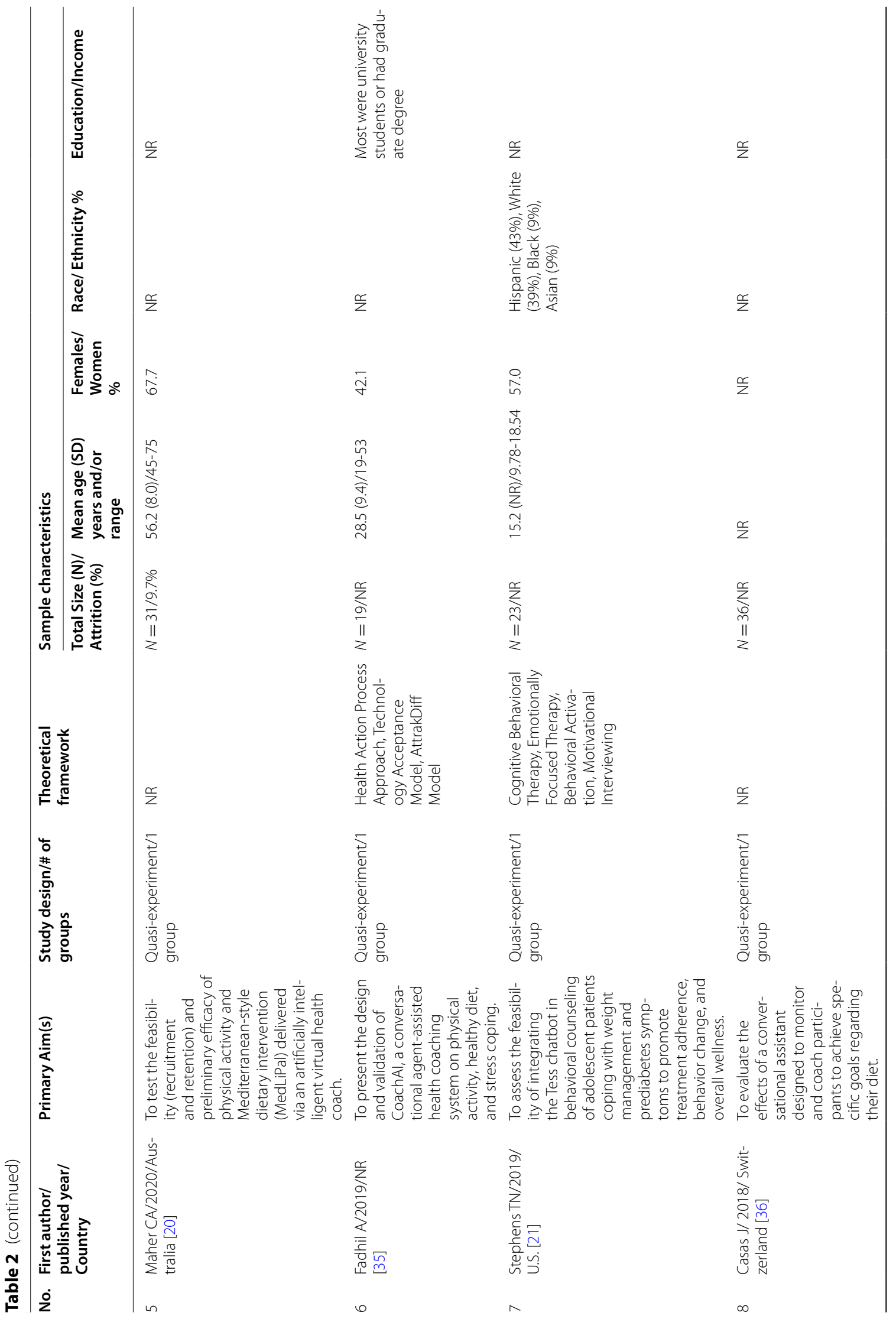




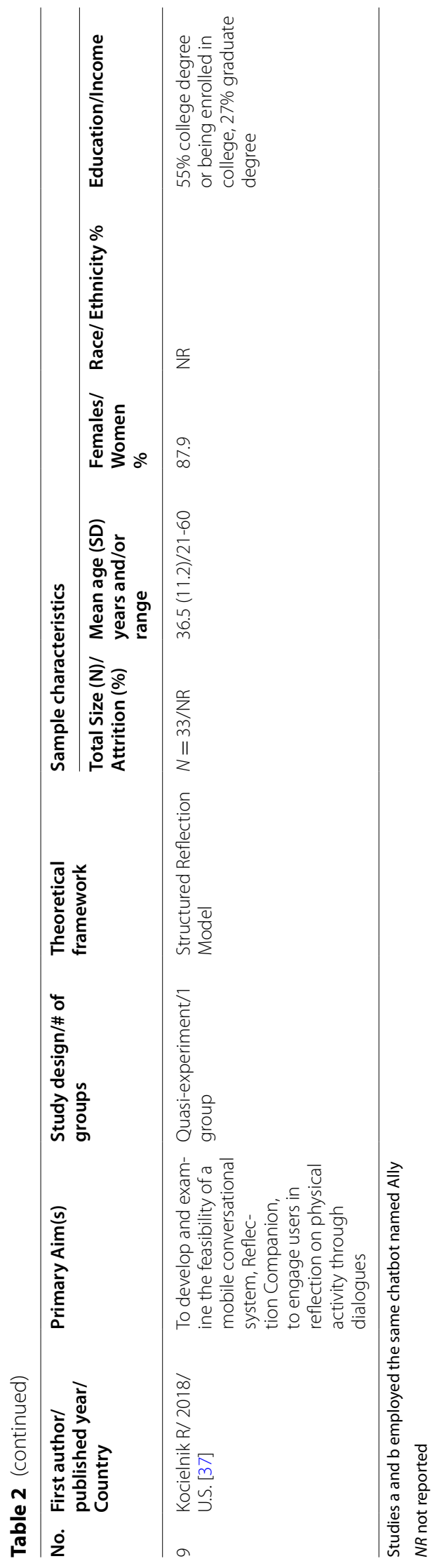


to 56.2 years $\left(\mathrm{SD}_{\text {range }}=2.0\right.$ to 13.7$)$, and females/women represented 42.1 to $87.9 \%$ of the sample. One study [21] solely targeted an adolescent population, whereas most studies targeted an adult population [20,31-35, 37]. One study [36] did not report the target population's age. Participants' race/ethnicity information was not reported in 8 out of the 9 studies. The study [21] that reported participants' race/ethnicity information included $43 \%$ Hispanic, 39\% White, 9\% Black, and 9\% Asian participants. Participants' education and income backgrounds were not reported in 5 out of the 9 studies. Among the 4 studies [31, 34, 35, 37] that reported the information, the majority included undergraduate students or people with graduate degrees. Overall, reporting of participants' sociodemographic information was inconsistent and insufficient across the studies.

Five studies employed quasi-experimental designs [20, $21,35-37$ ], and 4 were RCTs [31-34]. Only 5 studies [21, $31,32,35,37]$ used at least one theoretical framework. One was guided by 3 theories [35] and another by 4 theories [21]. The theories used in the 5 studies included the Health Action Process Approach $(n=2)$, the Habit Formation Model $(n=1)$, the Technology Acceptance Model $(n=1)$, the AttrakDiff Model $(n=1)$, Cognitive Behavioral Therapy $(n=1)$, Emotionally Focused Therapy $(n=1)$, Behavioral Activation $(n=1)$, Motivational Interviewing $(n=1)$, and the Structured Reflection Model $(n=1)$. It is notable that most of these theories were used to design the intervention contents for inducing behavioral changes. Only the Technology Acceptance Model and the AttrakDiff Model were relevant for guiding the designs of the chatbot characteristics and their technological platforms, independent from intervention contents.

\section{Summary of intervention and chatbot characteristics}

Figure 2 provides a visual summary of AI chatbot characteristics and intervention outcomes, and Table 3 provides more detailed information. The 9 studies varied in intervention and program length, lasting from 1 week to 3 months. For most studies $(n=8)$, the chatbot was the only intervention component for delivering contents and engaging with the participants. One study used multiintervention components, and participants had access to an AI chatbot along with a study website with educational materials [20]. A variety of commercially available technical platforms were used to host the chatbot and deliver the interventions, including Slack $(n=2)$, KakaoTalk $(n=1)$, Facebook messenger $(n=3)$, Telegram messenger $(n=1)$, WhatsApp $(n=1)$, and short messaging services (SMS) $(n=2)$. One study used 4 different platforms to deliver the intervention [21], and 2 studies used a chatbot app (i.e., Ally app) that was available on both Android and iOS systems [31, 33].
Following the AI Chatbot Behavior Change Model [22], we extracted features of the chatbot and intervention characteristics (Table 3). Regarding chatbot characteristics, identity features, such as specific names $(n=8)$ [20, $21,31-33,35-37]$ and chatbot gender $(n=3)$ [20, 31, 33], were specified. Notably, the chatbot gender was woman in the 3 studies that reported it [20,31,33]. All 9 chatbots delivered messages in text format. In addition to text, 3 chatbots used graphs [31, 33, 37], 2 used images [32, 35], 1 used voice [21], and 1 used a combination of graphs, images, and videos [36].

In 5 studies, the chatbots were constrained (i.e., users could only select pre-programmed responses in the chat) [31, 33-36], and in 4, the chatbots were unconstrained (i.e., users could freely type or speak to the chatbot) [20, 21, 32, 37]. Six chatbots [31-34, 36, 37] delivered daily intervention messages to the study participants. One chatbot communicated only on a weekly basis [20], and 1 communicated daily, weekly, on weekends or weekdays or at a scheduled date and time [35]. One study did not specify when and how often the messages were delivered [21]. Only 3 chatbots [20,21,32] were available ondemand so that users could initiate conversation at any preferred time. Most chatbots were equipped with relational capacity $(n=8$; i.e., conversation strategy to establish, maintain, or enhance social relationships with users) and persuasive capacity $(n=9$; i.e., conversation strategy to change user's behaviors and behavioral determinants), meaning that the conversations were designed to induce behavioral changes while engaging with users socially. While only 1 study [21] documented data security, none of the studies provided information on participant safety or ethics (i.e., ethical principle or standards with which the chatbot is designed).

\section{Summary of outcome measures and changes in outcomes}

Figure 2 also illustrates the outcome measures and changes in the main and secondary outcomes reported in both RCTs and quasi-experimental studies. Among 7 studies that measured PA [20, 21, 31-33, 35, 37], 2 used objective measures [31, 33], 4 used self-reported measures [20, 21, 32, 35], and 1 used both [37]. Self-reported dietary intake was measured in 4 studies [20, 34-36]. Only 1 study assessed objective changes in weight in a research office visit [20]. Details of intervention outcomes, including direction of effects, statistical significance, and magnitude, are presented in Table 4.

Sample sizes of the 4 RCT studies ranged from 106 to 274 and a priori power analyses were reported in 3 [31, $32,34]$, which showed that the sample sizes had sufficient power for analyzing the specified outcomes. Of the 4. RCT studies [31-34], 3 reported PA outcomes using daily step count $[31,33]$ and a self-reported habit index 


\begin{tabular}{|c|c|c|c|c|c|c|c|c|c|c|}
\hline \multirow{2}{*}{\multicolumn{2}{|c|}{$\begin{array}{l}\text { Chatbot } \\
\text { characteristics }\end{array}$}} & \multicolumn{4}{|c|}{ RCT } & \multicolumn{5}{|c|}{ Quasi-experimental study } \\
\hline & & 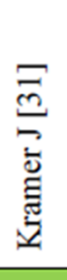 & 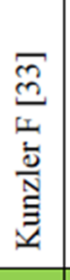 & 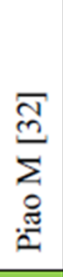 & 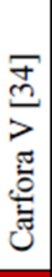 & 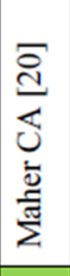 & 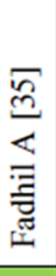 & 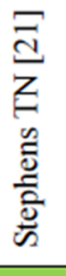 & 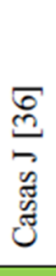 & 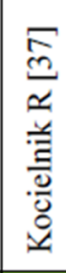 \\
\hline \multicolumn{11}{|l|}{ Name $^{\mathrm{a}}$} \\
\hline \multicolumn{2}{|c|}{ Gender $^{2}$} & & & & & & & & & \\
\hline \multirow{2}{*}{ Media $^{a}$} & Text-only & & & & & & & & & \\
\hline & Multiple media & & & & & & & & & \\
\hline \multirow{2}{*}{$\begin{array}{l}\text { User } \\
\text { input }^{\mathrm{a}}\end{array}$} & Constrained & & & & & & & & & \\
\hline & Unconstrained & & & & & & & & & \\
\hline \multicolumn{2}{|c|}{ Chatbot initiation $^{\mathrm{a}}$} & & & & & & & & & \\
\hline \multicolumn{2}{|c|}{ User initiation ${ }^{\mathrm{a}}$} & & & & & & & & & \\
\hline \multicolumn{2}{|c|}{ Relational capacity ${ }^{\mathrm{a}}$} & & & & & & & & & \\
\hline \multicolumn{2}{|c|}{ Persuasion capacity ${ }^{2}$} & & & & & & & & & \\
\hline \multirow{2}{*}{ Safety $^{3}$} & Data security & & & & & & & & & \\
\hline & $\begin{array}{l}\text { Participant } \\
\text { safety }\end{array}$ & & & & & & & & & \\
\hline \multicolumn{2}{|c|}{ Ethics discussion ${ }^{a}$} & & & & & & & & & \\
\hline \multicolumn{2}{|c|}{ Outcome measures } & & & & & & & & & \\
\hline \multicolumn{2}{|c|}{ Physical activity ${ }^{b}$} & + & + & + & & + & - & NR & & + \\
\hline \multicolumn{2}{|l|}{$\operatorname{Diet}^{b}$} & & & & + & + & - & & NR & \\
\hline \multicolumn{2}{|c|}{ Weight $^{\mathrm{b}}$} & & & & & + & & & & \\
\hline \multicolumn{2}{|c|}{ Engagement ${ }^{b}$} & NR & + & & & NR & & NR & & NR \\
\hline \multicolumn{2}{|c|}{$\begin{array}{l}\text { Acceptability/ } \\
\text { Satisfaction }^{b}\end{array}$} & & & & & & + & NR & NR & \\
\hline \multicolumn{2}{|c|}{ Adverse event ${ }^{b}$} & & & & & NR & & & & \\
\hline \multicolumn{2}{|l|}{ Other ${ }^{b}$} & & & & & NR & + & & & - \\
\hline
\end{tabular}

\begin{tabular}{|c|l|}
\hline & No (or not reported) (ab $^{\mathrm{ab}}$ \\
\hline+ & $\begin{array}{l}\text { Reported results were } \\
\text { statistically significant }\end{array}$ \\
\hline- & $\begin{array}{l}\text { Reported results were not } \\
\text { statistically significant }^{\mathrm{b}}\end{array}$ \\
\hline NR & $\begin{array}{l}\text { Reported results did not include } \\
\text { statistical significance }^{\mathrm{b}}\end{array}$ \\
\hline
\end{tabular}

Fig. 2 Summary of chatbot characteristics and intervention outcomes

[32]. In these RCTs, the AI chatbot intervention group resulted in a significant increase in PA, as compared to the control group, over the respective study period ( 6 weeks to 3 months). In terms of dietary change, 1 study [34] reported that participants in the intervention group showed higher self-reported intention to reduce red and processed meat consumption compared to the control group during a 2-week period.

In contrast, sample sizes for the 5 quasi-experimental studies were small, ranging from 19 to 36 participants, suggesting that these studies may lack statistical power to detect potential intervention effects. Among the 5 


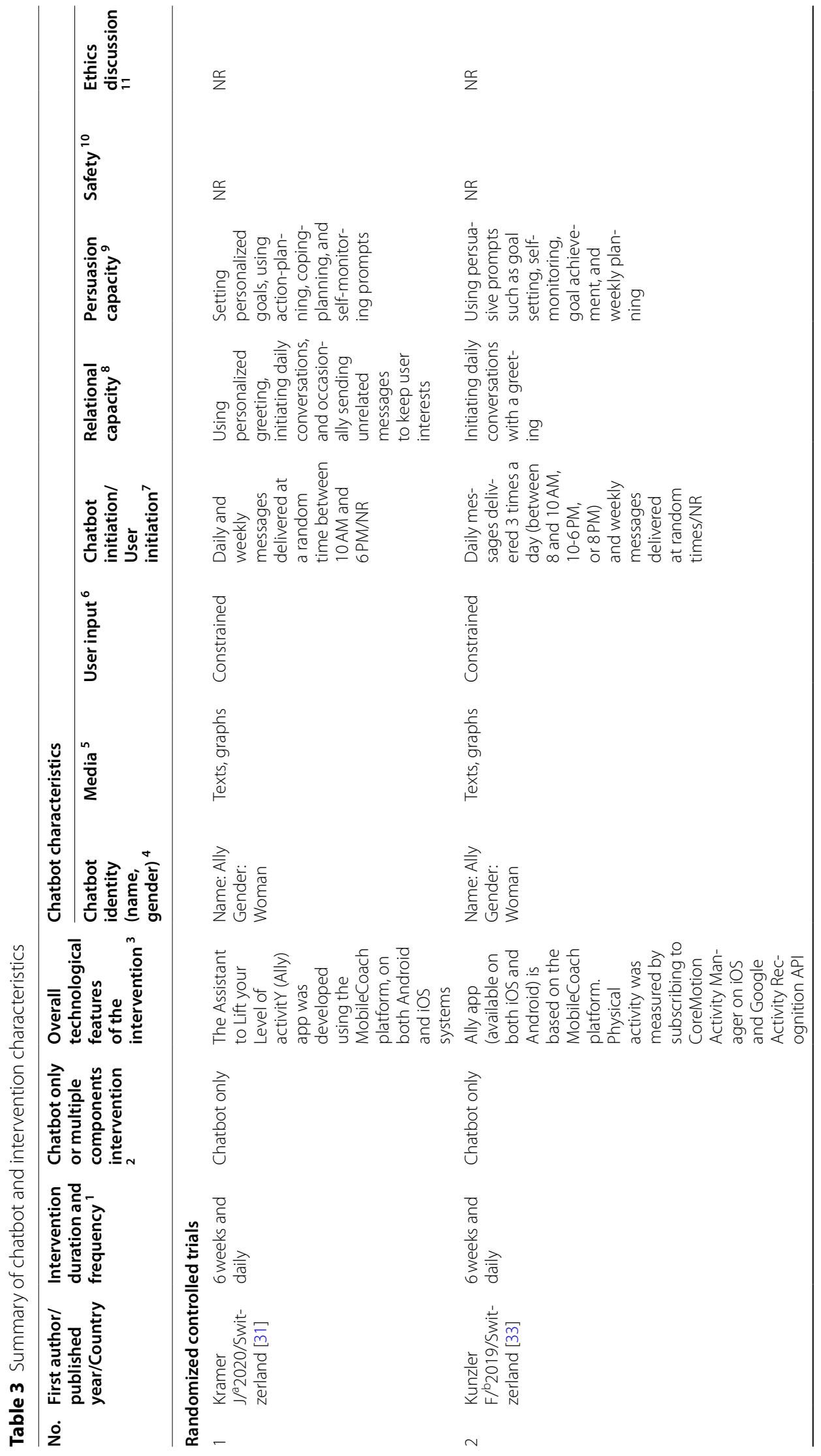




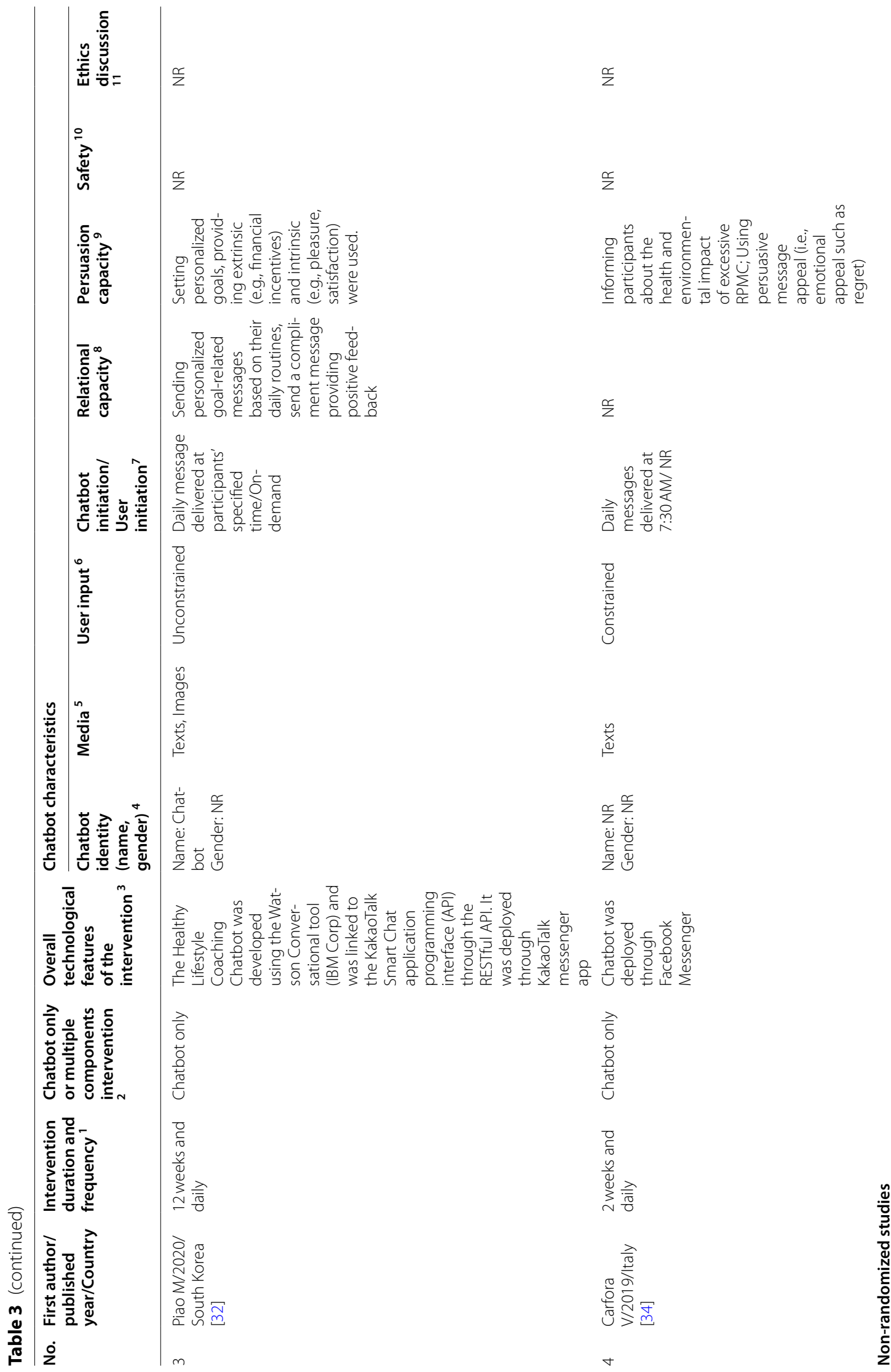




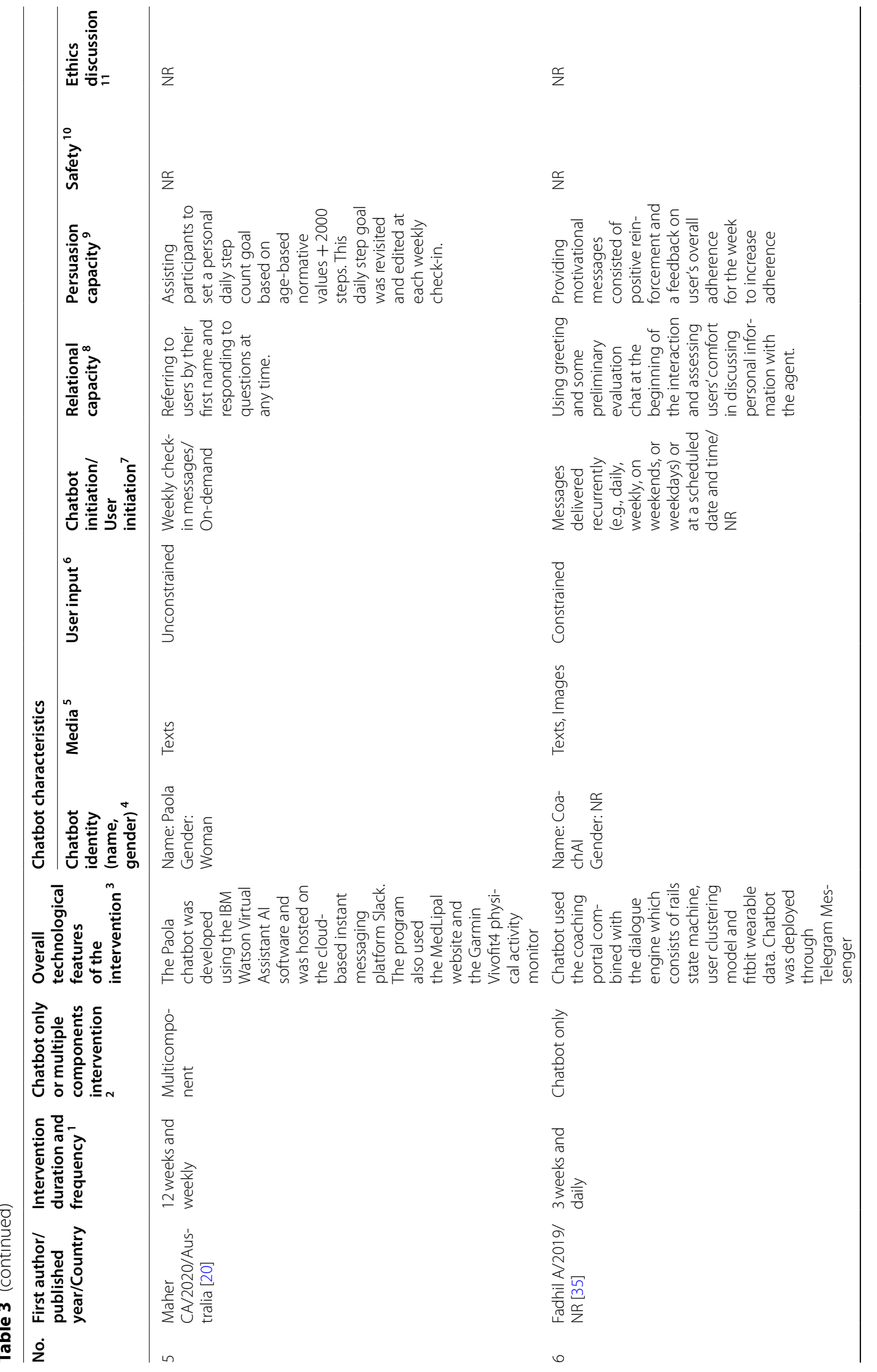




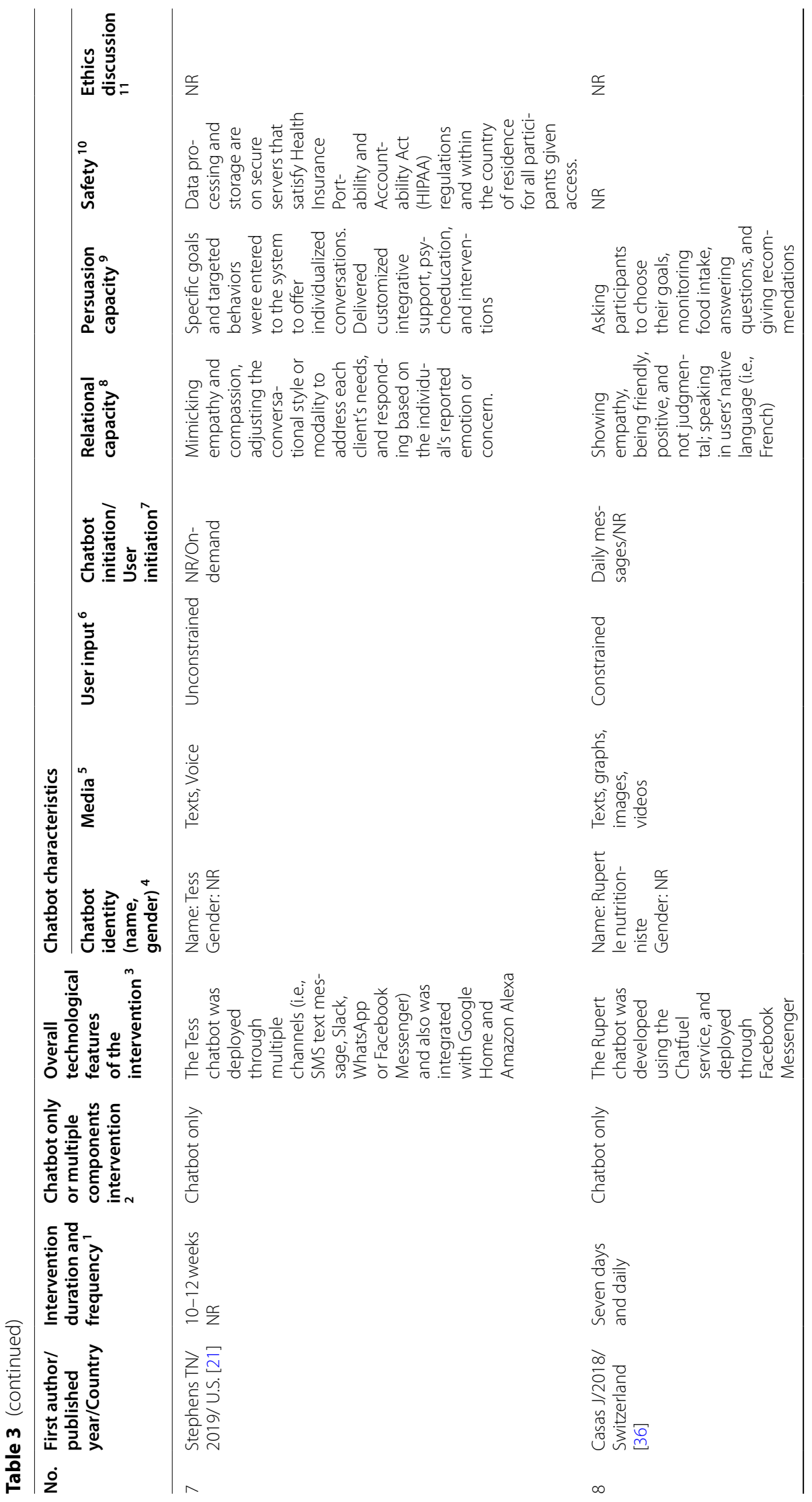




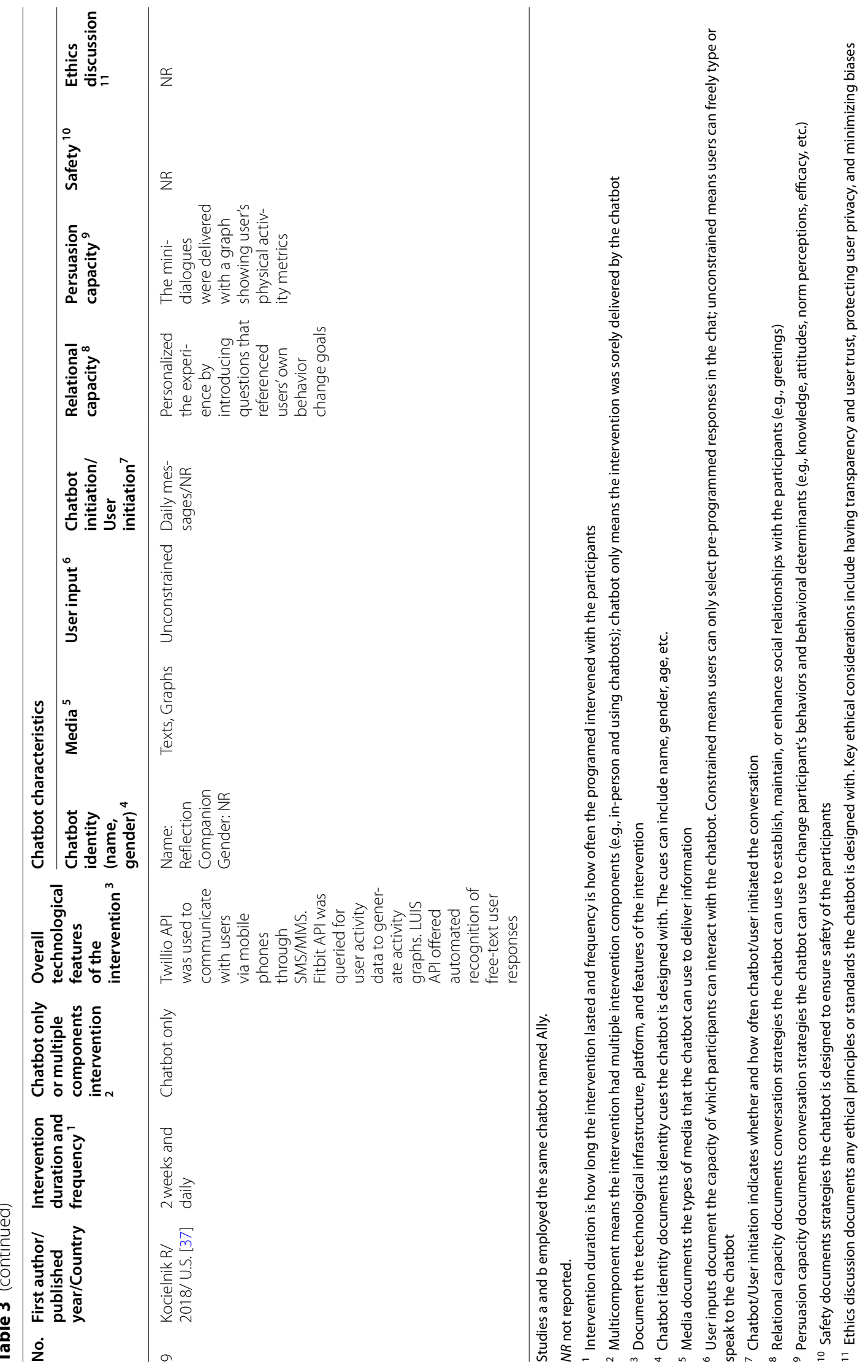


Oh et al. Int J Behave Nut Phys Act $\quad$ (2021) 18:160

Page 15 of 25

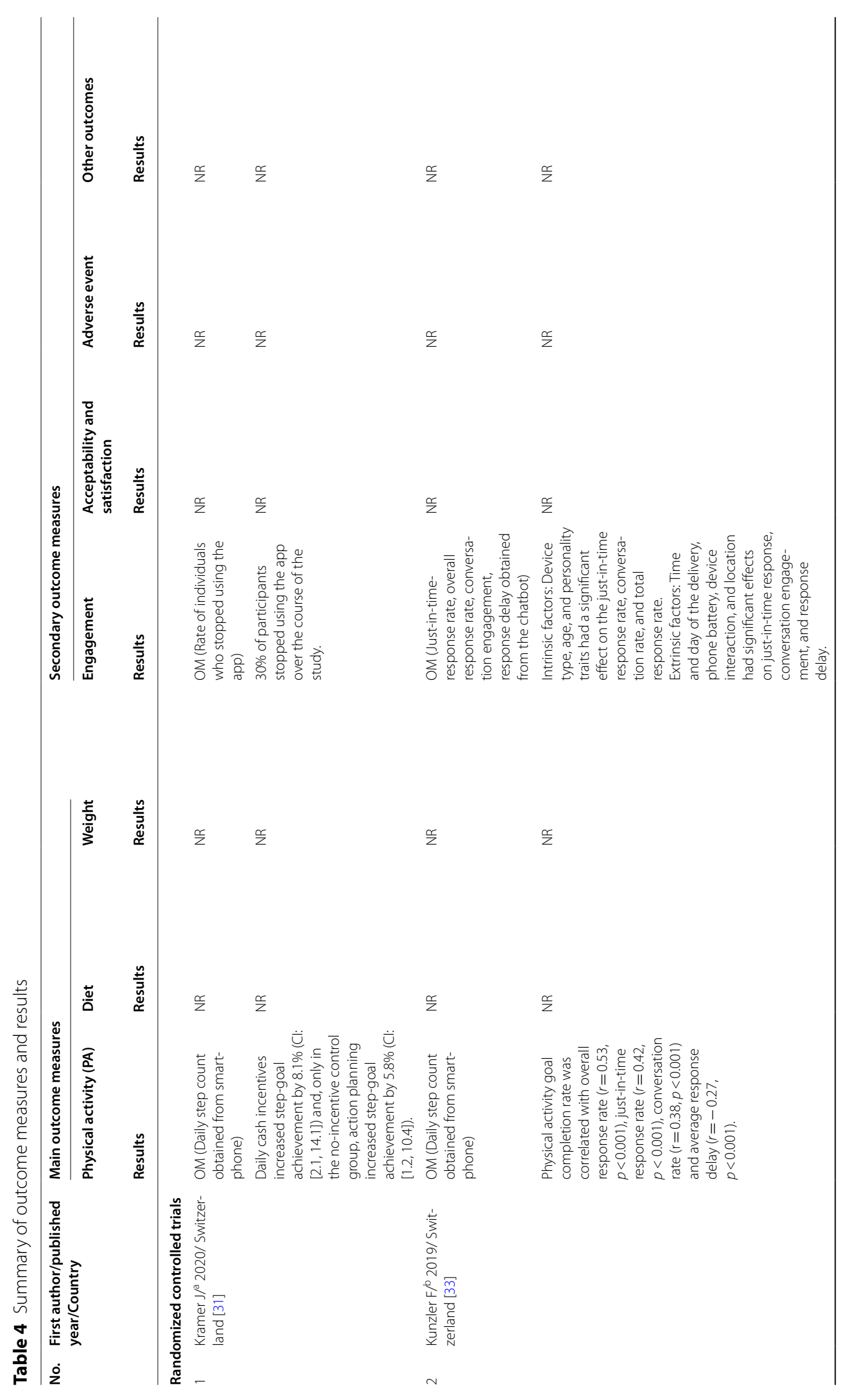




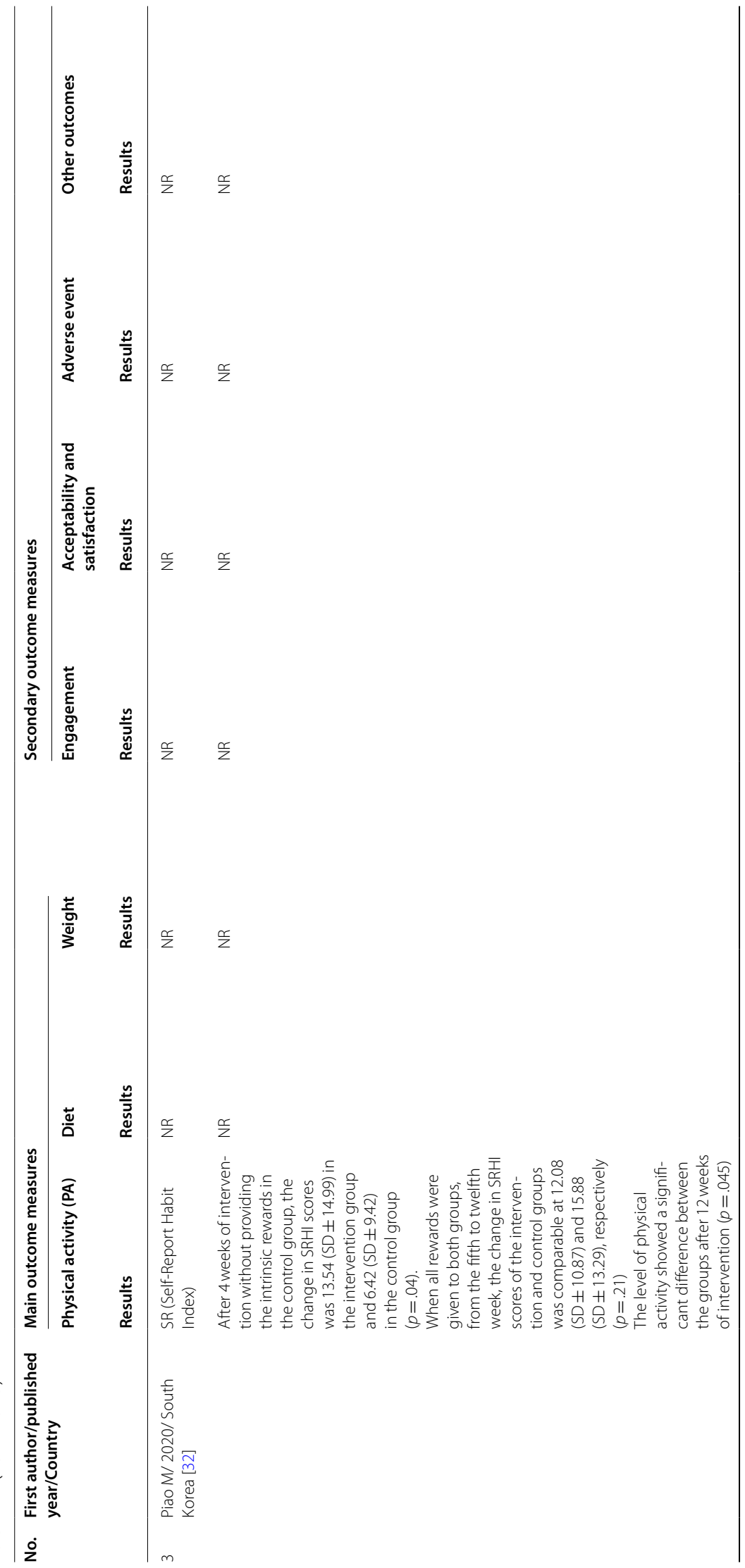


Oh et al. Int J Behav Nut Phys Act $\quad$ (2021) 18:160

Page 17 of 25

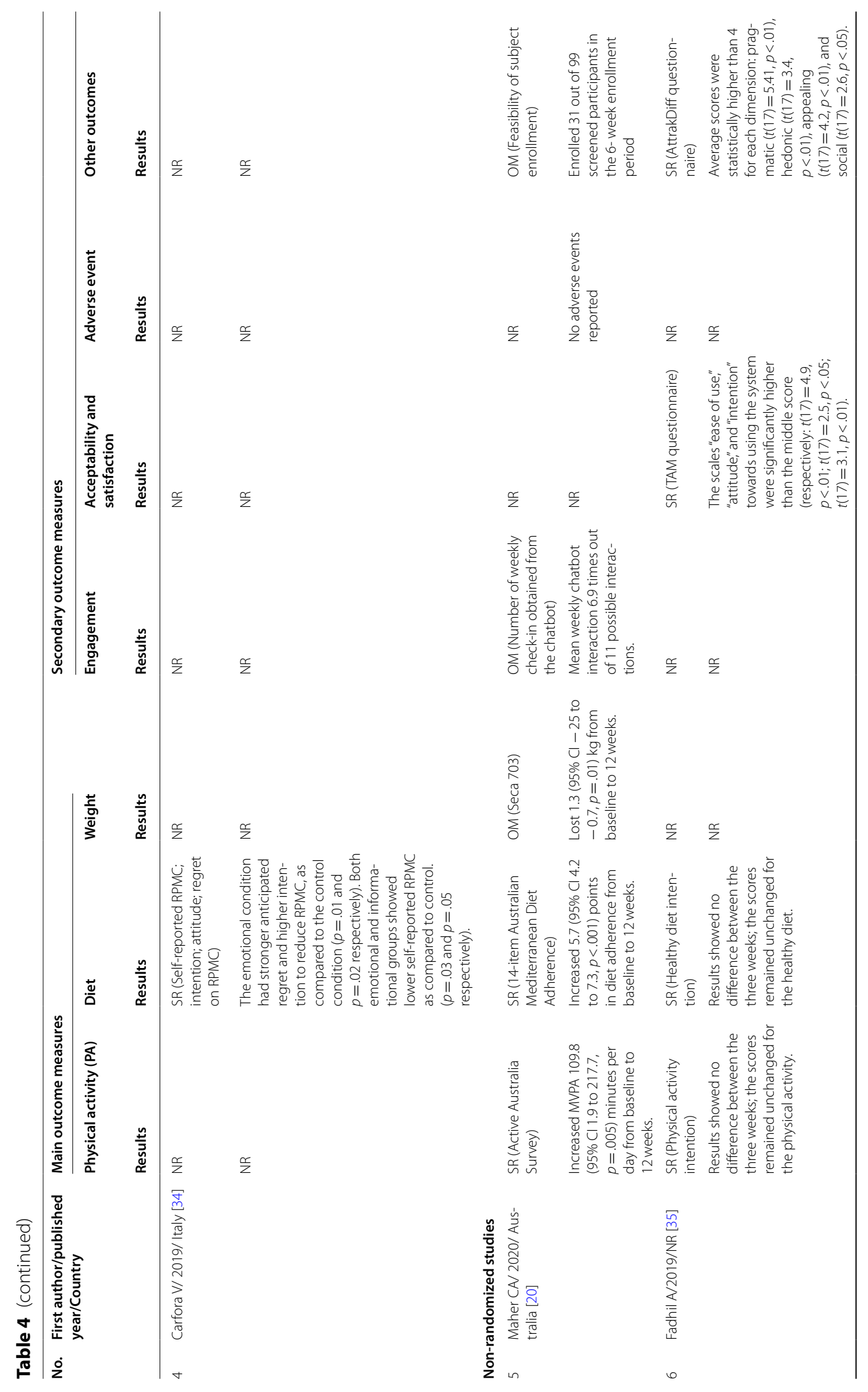




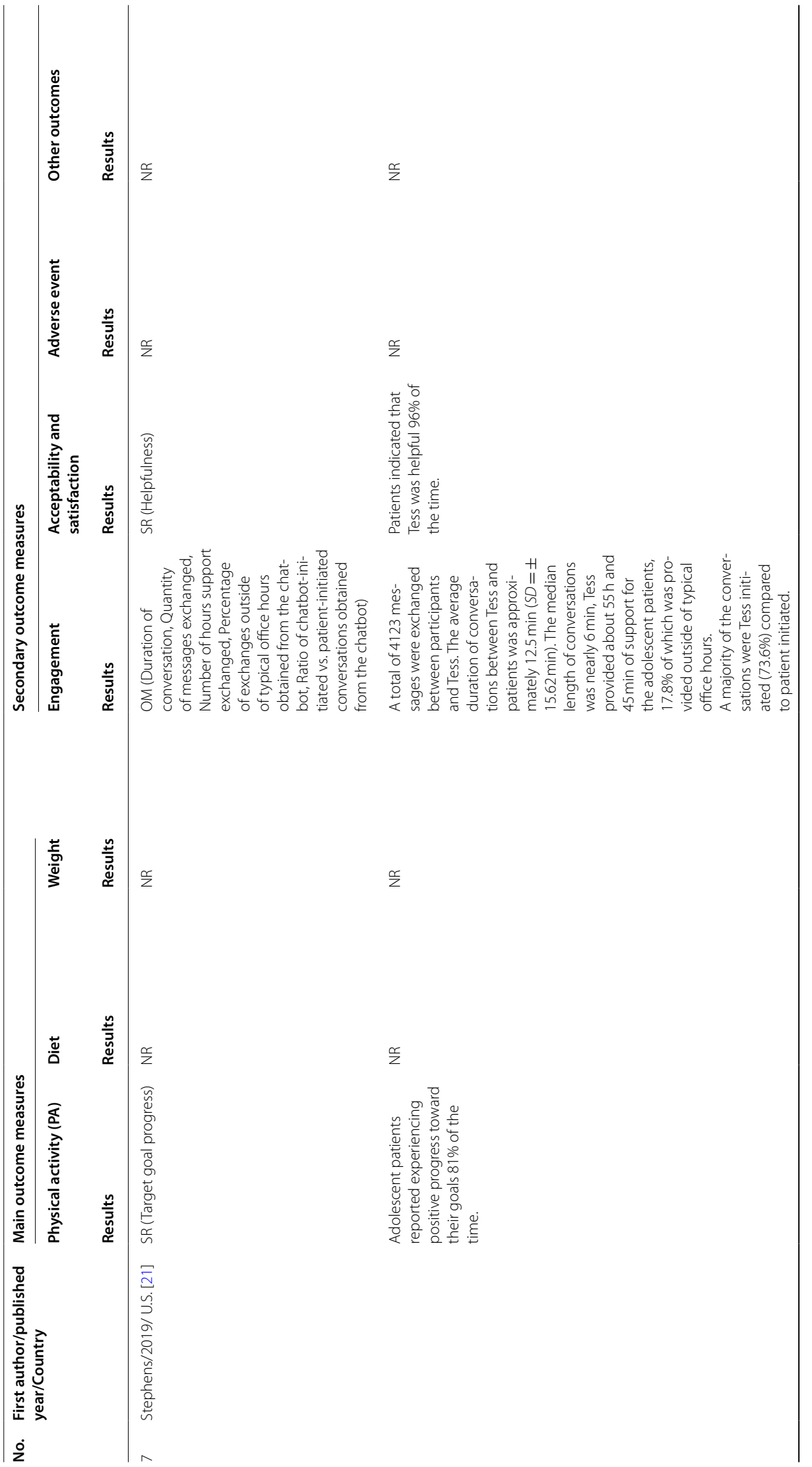




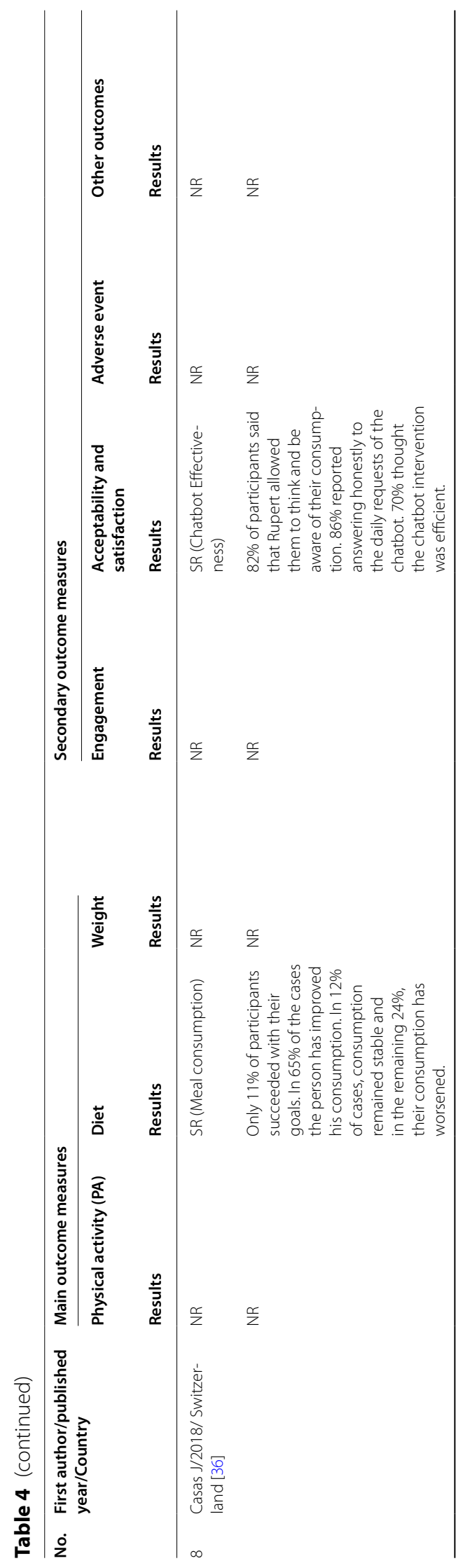




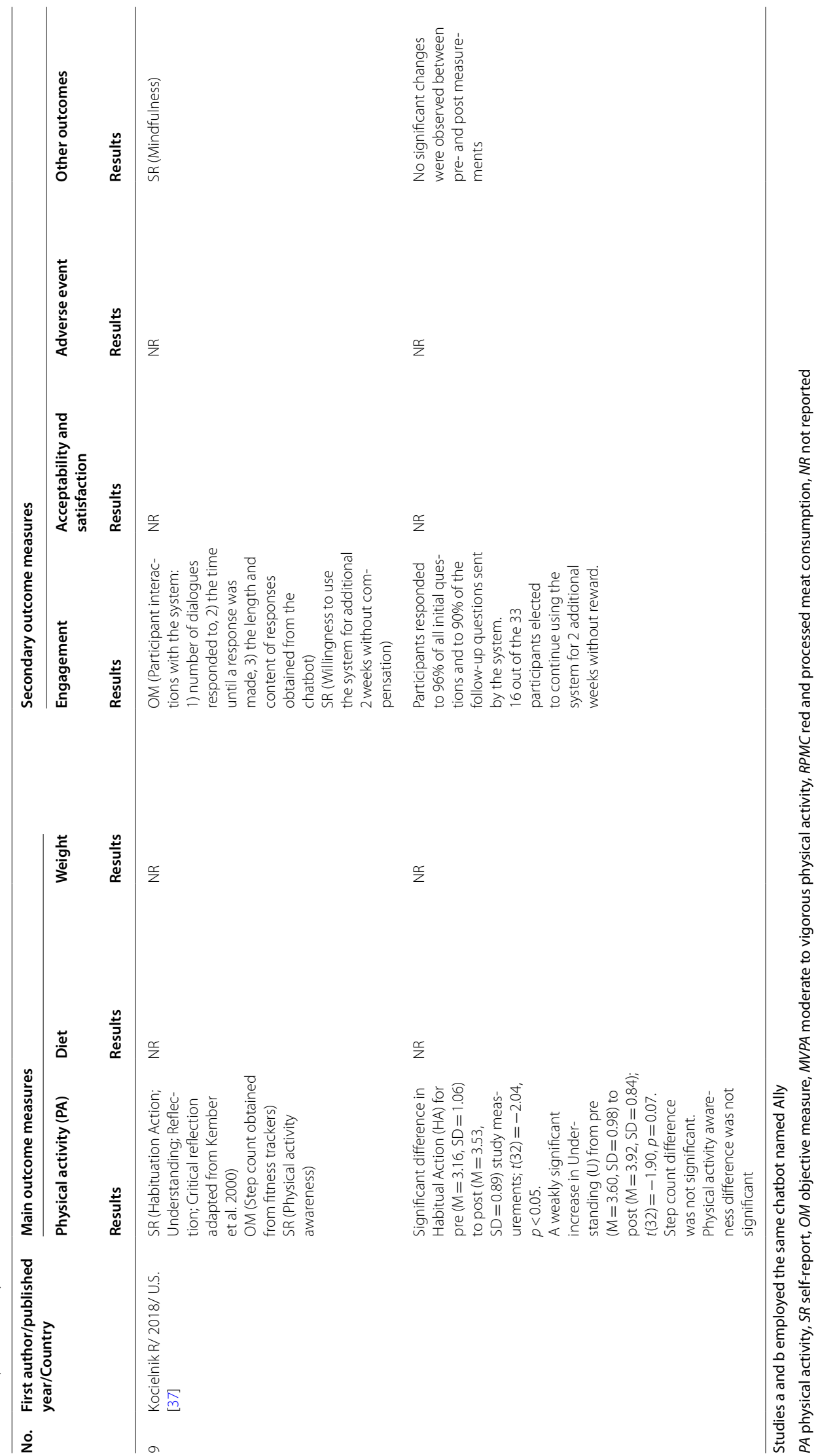


quasi-experimental studies, 2 [21, 37] reported only PA change outcomes, 1 [36] reported only diet change outcomes, and $2[20,35]$ reported both outcomes. With regard to PA-related outcomes, 2 studies reported statistically significant improvements [20,37]. Specifically, [20] observed increased moderate and vigorous PA over the study period [37]. found a significant increase in the habitual action of PA. One study [35] found no difference in PA intention within the intervention period. Although this study did not observe a statistically significant increase in PA intention, it revealed that among participants with either high or low intervention adherence, their PA intention showed an increasing trend over the study period [21]. only reported descriptive statistics and showed that participants experienced positive progress towards PA goals $81 \%$ of the time.

Among the quasi-experimental studies, only 1 study reported a statistically significant increase in diet adherence over 12 weeks [20] [35]. reported no difference of healthy diet intention over 3 weeks. In this study, participants with high intervention adherence showed a marginal increase, whereas, those with low adherence showed decreased healthy diet intention [36]. reported that participants' meal consumption improved in $65 \%$ of the cases. The only study [20] reporting pre-post weight change outcomes using objective weight measures showed that participants experienced a significant weight loss $(1.3 \mathrm{~kg})$ from baseline to 12 weeks. To summarize, non-significant findings and a lack of statistical reporting were more prevalent in the quasi-experimental studies, but the direction of intervention effects were similar to those reported in the RCTs.

Engagement, acceptability/satisfaction, and safety measures were reported as secondary outcomes in 7 studies [20, 21, 31, 33, 35-37]. Five studies reported engagement [20,21,31,33,37] using various types of measurements, such as user response rate to chatbot messages [31], frequency of users' weekly check-ins [20], and length of conversations between the chatbot and users [21]. Three studies measured acceptability/ satisfaction of the chatbot $[21,35,36]$ using measures such as technology acceptance [35], helpfulness of the chatbot [21], and perceived efficiency of chatbot communications [36]. Regarding reporting of adverse events (e.g., experiencing side effects from interventions), only 1 study reported that no adverse events related to study participation were experienced [20]. Three studies reported additional measures, including feasibility of subject enrollment [20], using the AttrakDiff questionnaire for measuring four aspects of the chatbot (i.e., pragmatic, hedonic, appealing, social) [35], and assessing perceived mindfulness about own behaviors [37].
Among 5 studies that reported engagement [20, 21, $31,33,37$ ], only 1 [33] reported statistical significance of the effects of intrinsic (e.g., age, personality traits) and extrinsic factors (e.g., time and day of the delivery, location) on user engagement (e.g., conversation engagement, response delay). Among 3 studies [21, 35, 36] that reported acceptability/satisfaction, 1 study [35] found that the acceptability of the chatbot was significantly higher than the middle score corresponding to "neutral" (i.e., 4 on a 7-point scale). One study that reported the safety of the intervention did not include statistical significance [20]. Three studies reported other measures [20, $35,37]$, and 1 found that pragmatic, hedonic, appealing, and social ratings of the chatbot were significantly higher than the middle score [35]. Another study [37] found no significant changes in the perceived mindfulness between pre- and post-study.

\section{Summary of quality assessment and risk of bias}

The results of risk of bias assessments of the 9 studies are reported in Additional file 2. Of the 4 RCT studies [31-34], 3 were rated as fair [31,32,34] and 1 was rated as poor [33] due to its lack of reporting of several critical. The poorly rated study did not report overall dropout rates or the differential dropout rates between treatment groups, did not report that the sample size was sufficiently large to be able to detect differences between groups (i.e., no power analysis), and did not prespecify outcomes for hypothesis testing. Of the 5 quasiexperimental studies [20,21,35-37], 1 study was rated as fair [20] and 4 studies were rated as poor [21,35-37] due to flaws with regard to several critical. These studies reported neither a power analysis to ensure that the sample size was sufficiently large, nor follow-up rates after baseline. Additionally, the statistical methods did not examine pre-to-post changes in outcome measures and lacked reporting of statistical significance.

\section{Discussion}

This systematic review aimed to evaluate the characteristics and potential efficacy of AI chatbot interventions to promote PA, healthy diet, and/or weight management. Most studies focused on changes in PA, and majority $[20,31-33,37]$ reported significant improvements in PA-related behaviors. The number of studies with the aim to change diet and weight status was small. Two studies $[20,34]$ found significant improvements in dietrelated behaviors. Although only 1 study [20] reported weight-related outcomes, it reported significant weight change after the intervention. In summation, chatbots can improve PA, but the study not able to make definitive 
conclusions on the potential efficacy of chatbot interventions on promoting PA, healthy eating, or weight loss.

This qualitative synthesis of effects needs to be interpreted with caution given that the reviewed studies lack consistent usage of measurements and reporting of outcome evaluations. These studies used different measurements and statistical methods to evaluate PA and diet outcomes. For example, 1 study [20] measured one's self-reported change in MVPA during the intervention period to gauge the efficacy of the intervention, whereas in another study [31] step-goal achievement was used as a measure of the intervention efficacy. The two quasi-experimental studies did not report statistical significance of the pre-post changes in PA or diet outcomes $[21,36]$. Such inconsistency in evaluating the potential efficacy of interventions has been reported in previous systematic reviews $[1,38]$. To advance the application of chatbot interventions in lifestyle modification programs and to demonstrate the rigor of their efficacy, future studies should examine multiple behavior change indicators, ideally incorporating objectively measured outcomes.

Consistent with other systematic reviews of chatbot interventions in health care and mental health [1,38], reporting of participants' engagement, acceptability/satisfaction, and adverse events was limited in the studies. In particular, engagement, acceptability, and satisfaction measures varied across the studies, impeding the systematic summarization and assessment of various intervention implementations. For instance, 1 study [33] used user response rates and user response delay as engagement measures, whereas in another study [21], the duration of conversation and the ratio of chatbot-initiated on patient-initiated conversations were used to assess the level of user engagement. Inconsistent reporting of user engagement, acceptability, and satisfaction measures may be problematic because it could contribute challenges to the interpretation and comparison of the results across different chatbot systems [1]. Therefore, standardization of these measures should be implemented in future research. For example, as suggested in previous studies $[39,40]$, conversational turns per session can be a viable, objective, and quantitative metric for user engagement. Regarding reporting of adverse events, despite the recommendation of reporting adverse events in clinical trials by the Consolidated Standards of Reporting Trials Group [41], only 1 study [20] reported adverse events. It is recommended that future studies consistently assess and report any unexpected events resulting from the use of AI chatbots to prevent any side effects or potential harm to participants.

Theoretical frameworks for designing and evaluating a chatbot system are essential to understand the rationale behind participants' motivation, engagement, and behaviors. However, theoretical frameworks were not reported in many of the studies included in this systematic review. The lack of theoretical foundations of existing chatbot systems has also been noted in previous literature [42]. In this review, we found that the majority of AI chatbots were equipped with persuasion strategies (e.g., setting personalized goals) and relational strategies (e.g., showing empathy) to establish, maintain, or enhance social relationships with participants. The application of theoretical frameworks will guide in developing effective communicative strategies that can be implemented into chatbot designs. For example, designing chatbots with personalized messages can be more effective than non-tailored and standardized messages $[43,44]$. For relational strategies, future studies can benefit from drawing on the literature on humancomputer interaction and relational agents (e.g., [45, 46]) and interpersonal communication theories (e.g., Social Penetration Theory [47]) to develop strategies to facilitate relation formation between participants and chatbots.

Regarding designs of chatbot characteristics and dialogue systems, the rationale behind using human-like identity features (e.g., gender selection) on chatbots was rarely discussed. Only 1 study [31] referred to literature on human-computer interaction [48] and discussed the importance of using human-like identity features on chatbots to facilitate successful human-chatbot relationships. Additionally, only one chatbot [21] was able to deliver spoken outputs. This is inconsistent with a previous systematic review on chatbots used in health care, in which spoken chatbot output was identified as the most common delivery mode across the studies [1].

With regard to user input, over half of the studies [31, 33-36] used a constrained AI chatbot, while the remaining $[20,21,32,37]$ used unconstrained AI chatbots. Constrained AI chatbots are rule-based, well-structured, and easy to build, control, and implement, thus ensuring the quality and consistency in the structure and delivery of content [42]. However, they are not able to adapt to participants' inquiries and address emergent questions, and are, thus, not suitable for sustaining more natural and complex interactions with participants [42]. In contrast, unconstrained AI chatbots are known to simulate naturalistic human-to-human communication and may strengthen interventions in general, particularly in the long-term, due to their flexibility and adaptability in conversations $[1,38,42]$. With increasing access to large health care datasets, advanced technologies [49], and new developments in machine learning that allow for complex dialogue management methods and conversational flexibility [1], employing unconstrained chatbots to yield long-term efficacy may become more feasible in 
future research. For instance, increasing the precision of natural language understanding and generation will allow for AI chatbots to better engage users in conversations and follow up with tailored intervention messages.

Safety and data security criteria are essential in designing chatbots. However, only 1 study provided descriptions of these criteria. Conversations between study participants and chatbots should be carefully monitored since erroneous chatbot responses may result in unintended harm. In particular, as conversational flexibility increases, there may be an increase in potential errors associated with natural language understanding or response generation [1]. Thus, using unconstrained chatbots should be accompanied with careful monitoring of participant and chatbot interactions, and of safety functions.

\section{Strengths and limitations}

This review has several strengths. First, to the best of our knowledge, this is the first review to systematically examine the characteristics and potential efficacy of AI chatbot interventions in lifestyle modifications, thereby providing crucial insights for identifying gaps and future directions for research and clinical practice. Second, we developed comprehensive search strategies with an MLS for six electronic databases to increase the sensitivity and comprehensiveness of our search. Despite its strengths, several limitations need to also be acknowledged. First, we did not search gray literature in this systematic review. Second, we limited our search to peer-reviewed studies published as full-text in English only. Lastly, due to the heterogeneity of outcome measures and the limited number of RCT designs in this systematic review, we were not able to conduct a meta-analysis and make firm conclusions of the potential efficacy of chatbot interventions. In addition, the small sample sizes used by the studies made it difficult to scale the results to general populations. More RCTs with larger sample sizes and longer study durations are needed to determine the efficacy of AI chatbot interventions on improving PA, diet, and weight loss.

\section{Conclusions}

AI chatbot technologies and their commercial applications continue to rapidly develop, as do the number of studies about these technologies. Chatbots may improve PA, but this study was not able to make definitive conclusions of the potential efficacy of chatbot interventions on PA, diet, and weight management/ loss. Despite the rapid increase in publications about chatbot designs and interventions, standard measures for evaluating chatbot interventions and theory-guided chatbots are still lacking. Thus, there is a need for future studies to use standardized criteria for evaluating chatbot implementation and efficacy. Additionally, theoretical frameworks that can capture the unique factors of human-chatbot interactions for behavior changes need to be developed and used to guide future AI chatbot interventions. Lastly, as increased adoption of chatbots will be expected for diverse populations, future research needs to consider equity and equality in designing and implementing chatbot interventions. For target populations with different sociodemographic backgrounds (e.g., living environment, race/ethnicity, cultural backgrounds, etc.), specifically tailored designs and sub-group evaluations need to be employed to ensure adequate delivery and optimal intervention impact.

\section{Abbreviations}

Al: Artificial Intelligence; PROSPERO: International Prospective Register of Systematic Reviews; RCT: Randomized controlled trial; PA: Physical activity; MLS: Medical librarian.

\section{Supplementary Information}

The online version contains supplementary material available at https://doi. org/10.1186/s12966-021-01224-6.

Additional file 1. Search strategies for PubMed, EMBASE, ACM Digital Library, Web of Science, PsycINFO, and IEEE.

Additional file 2. Summary of quality assessment and risk of bias.

\section{Acknowledgements}

Not applicable.

\section{Authors' contributions}

YF, JZ, and YO contributed to the conception and design of the review; MF and $Y O$ developed the search strategies; $Y F, J Z$, and $Y O$ contributed to the screening of papers and synthesizing the results into tables; YF, JZ, and YO wrote sections of the systematic review. All authors contributed to manuscript revision, read, and approved the submitted version. YF is the guarantor of the review.

\section{Funding}

This project was supported by a grant (K24NR015812) from the National Institute of Nursing Research (Dr. Fukuoka) and the Team Science Award by the University of California, San Francisco Academic Senate Committee on Research. Publication made possible in part by support from the UCSF Open Access Publishing Fund. The study sponsors had no role in the study design; collection, analysis, or interpretation of data; writing the report; or the decision to submit the report for publication.

Availability of data and materials Not applicable.

\section{Declarations}

Ethics approval and consent to participate Not applicable.

Consent for publication

Not applicable. 


\section{Competing interests}

The authors declare that they have no competing interests.

\section{Author details}

${ }^{1}$ Department of Communication, University of California Davis, Davis, USA. ${ }^{2}$ Department of Public Health Sciences, University of California Davis, Davis, USA. ${ }^{3}$ Education and Research Services, University of California, San Francisco (UCSF) Library, UCSF, San Francisco, USA. ${ }^{4}$ Department of Physiological Nursing, UCSF, San Francisco, USA.

Received: 31 May 2021 Accepted: 10 November 2021

Published online: 11 December 2021

\section{References}

1. Laranjo L, Dunn AG, Tong HL, Kocaballi AB, Chen J, Bashir R, et al. Conversational agents in healthcare: a systematic review. J Am Med Inform Assoc. 2018;25(9):1248-58.

2. Pew Research Center. Nearly half of Americans use digital vocie assistants, mostly on their smartphones 2017. Available from: https://www.pewre search.org/fact-tank/2017/12/12/nearly-half-of-americans-use-digitalvoice-assistants-mostly-on-their-smartphones/.

3. Farhud DD. Impact of lifestyle on health. Iran J Public Health. 2015:44(11):1442.

4. Cecchini M, Sassi F, Lauer JA, Lee YY, Guajardo-Barron V, Chisholm D. Tackling of unhealthy diets, physical inactivity, and obesity: health effects and cost-effectiveness. Lancet. 2010;376(9754):1775-84.

5. Wagner $\mathrm{K}-\mathrm{H}$, Brath $\mathrm{H}$. A global view on the development of non communicable diseases. Prev Med. 2012;54:S38-41.

6. Bennett JE, Stevens GA, Mathers CD, Bonita R, Rehm J, Kruk ME, et al. NCD countdown 2030: worldwide trends in non-communicable disease mortality and progress towards sustainable development goal target 3.4. Lancet. 2018;392(10152):1072-88.

7. Clarke T, Norris T, Schiller JS. Early release of selected estimates based on data from the National Health Interview Survey. Natl Center Health Stat. 2019.

8. Department of Health and Human Services. Physical Activity Guidelines for Americans, 2nd edition. Washington, DC: U.S. Department of Health and Human Services; 2018. Available from: https://health.gov/sites/defau It/files/2019-09/Physical_Activity_Guidelines_2nd_edition.pdf.

9. Hales C, Carroll M, Fryar C, Ogden C. Prevalence of obesity and severe obesity among adults: United States, 2017-2018. NCHS Data Brief, no 360 Hyattsville: National Center for Health Statistics; 2020.

10. Prentice AM. The emerging epidemic of obesity in developing countries. Int J Epidemiol. 2006:35(1):93-9.

11. Vandelanotte C, Müller AM, Short CE, Hingle M, Nathan N, Williams $\mathrm{SL}$, et al. Past, present, and future of eHealth and $\mathrm{mHealth}$ research to improve physical activity and dietary behaviors. J Nutr Educ Behav. 2016;48(3):219-28. e1

12. Case MA, Burwick HA, Volpp KG, Patel MS. Accuracy of smartphone applications and wearable devices for tracking physical activity data. Jama. 2015:313(6):625-6.

13. Zhang J, Brackbill D, Yang S, Becker J, Herbert N, Centola D. Support or competition? How online social networks increase physical activity: a randomized controlled trial. Prev Med Rep. 2016:4:453-8.

14. Zhang J, Brackbill D, Yang S, Centola D. Efficacy and causal mechanism of an online social media intervention to increase physical activity: results of a randomized controlled trial. Prev Med Rep. 2015;2:651-7.

15. Mateo GF, Granado-Font E, Ferré-Grau C, Montaña-Carreras X. Mobile phone apps to promote weight loss and increase physical activity: a systematic review and meta-analysis. J Med Internet Res. 2015;17(11):e253.

16. Manzoni GM, Pagnini F, Corti S, Molinari E, Castelnuovo G. Internet-based behavioral interventions for obesity: an updated systematic review. Clin Pract Epidemiol Ment Health. 2011;7:19.

17. Beleigoli AM, Andrade AQ, Cançado AG, Paulo MN, Maria De Fátima HD, Ribeiro AL. Web-based digital health interventions for weight loss and lifestyle habit changes in overweight and obese adults: systematic review and meta-analysis. J Med Internet Res. 2019;21(1):e298.

18. Laranjo L, Arguel A, Neves AL, Gallagher AM, Kaplan R, Mortimer N, et al. The influence of social networking sites on health behavior change: a systematic review and meta-analysis. J Am Med Inform Assoc. 2015;22(1):243-56.

19. Laranjo L, Ding D, Heleno B, Kocaballi B, Quiroz JC, Tong HL, et al. Do smartphone applications and activity trackers increase physical activity in adults? Systematic review, meta-analysis and metaregression. Br J Sports Med. 2021;55(8):422-32.

20. Maher CA, Davis CR, Curtis RG, Short CE, Murphy KJ. A physical activity and diet program delivered by artificially intelligent virtual health coach: proof-of-concept study. JMIR mHealth uHealth. 2020;8(7):e17558.

21. Stephens TN, Joerin A, Rauws M, Werk LN. Feasibility of pediatric obesity and prediabetes treatment support through Tess, the Al behavioral coaching chatbot. Transl Behav Med. 2019;9(3):440-7.

22. Zhang J, Oh YJ, Lange P, Yu Z, Fukuoka Y. Artificial intelligence Chatbot behavior change model for designing artificial intelligence Chatbots to promote physical activity and a healthy diet. J Med Internet Res. 2020;22(9):e22845.

23. Pereira J, Díaz Ó. Using health chatbots for behavior change: a mapping study. J Med Syst. 2019;43(5):135.

24. Miner AS, Laranjo L, Kocaballi AB. Chatbots in the fight against the COVID-19 pandemic. NPJ Digit Med. 2020;3(1):1-4

25. Gentner T, Neitzel T, Schulze J, Buettner R. A Systematic literature review of medical chatbot research from a behavior change perspective. In 2020 IEEE 44th Annual Computers, Software, and Applications Conference (COMPSAC). IEEE; 2020. p. 735-40.

26. Vaidyam AN, Wisniewski H, Halamka JD, Kashavan MS, Torous JB. Chatbots and conversational agents in mental health: a review of the psychiatric landscape. Can J Psychiatry. 2019;64(7):456-64

27. Schardt C, Adams MB, Owens T, Keitz S, Fontelo P. Utilization of the PICO framework to improve searching PubMed for clinical questions. BMC Med Inform Decis Making. 2007;7(1):1-6.

28. Clarivate Analytics. Endnote x9 2019. Available from: https://endnote. $\mathrm{com} /$.

29. Covidence systematic review software. Melbourne, Australia: Veritas Health Innovation. Available from: www.covidence.org.

30. NIH National Heart, Lung, and Blood Institute. Study quality assessment tools. Available from: https://www.nhlbi.nih.gov/health-topics/studyquality-assessment-tools.

31. Kramer J-N, Künzler F, Mishra V, Smith SN, Kotz D, Scholz U, et al. Which components of a smartphone walking app help users to reach personalized step goals? Results from an optimization trial. Ann Behav Med. 2020;54(7):518-28.

32. Piao M, Ryu H, Lee H, Kim J. Use of the healthy lifestyle coaching Chatbot app to promote stair-climbing habits among office workers: exploratory randomized controlled trial. JMIR mHealth uHealth. 2020;8(5):e15085.

33. Künzler F, Mishra V, Kramer J-N, Kotz D, Fleisch E, Kowatsch T. Exploring the state-of-receptivity for mhealth interventions. Proc ACM Interact Mobile Wearable Ubiquitous Technol. 2019:3(4):1-27.

34. Carfora V, Bertolotti M, Catellani P. Informational and emotional daily messages to reduce red and processed meat consumption. Appetite. 2019;141:104331.

35. Fadhil A, Wang Y, Reiterer $\mathrm{H}$. Assistive conversational agent for health coaching: a validation study. Methods Inf Med. 2019;58(1):009-23.

36. Casas J, Mugellini E, Khaled OA editors. Food diary coaching chatbot. Proceedings of the 2018 ACM International Joint Conference and 2018 International Symposium on Pervasive and Ubiquitous Computing and Wearable Computers; 2018

37. Kocielnik R, Xiao L, Avrahami D, Hsieh G. Reflection companion: a conversational system for engaging users in reflection on physical activity. Proc ACM Interact Mobile Wearable Ubiquitous Technol. 2018;2(2):1-26.

38. Milne-Ives M, de Cock C, Lim E, Shehadeh MH, de Pennington $N$, Mole $\mathrm{G}$, et al. The effectiveness of artificial intelligence conversational agents in health care: systematic review. J Med Internet Res. 2020;22(10):e20346.

39. Abd-Alrazaq A, Safi Z, Alajlani M, Warren J, Househ M, Denecke K. Technical metrics used to evaluate health care Chatbots: scoping review. J Med Internet Res. 2020:22(6):e18301.

40. Shum H-Y, He X-d, Li D. From Eliza to Xiaolce: challenges and opportunities with social chatbots. Frontiers of Information Technology \& Electronic. Engineering. 2018;19(1):10-26.

41. Moher D, Hopewell S, Schulz KF, Montori V, Gøtzsche PC, Devereaux P, et al. CONSORT 2010 explanation and elaboration: updated guidelines 
for reporting parallel group randomised trials. J Clin Epidemiol. 2010;63(8):e1-e37.

42. Fadhil A. Can a chatbot determine my diet?: Addressing challenges of chatbot application for meal recommendation. arXiv preprint arXiv:180209100. 2018.

43. Kreuter MW, Wray RJ. Tailored and targeted health communication: strategies for enhancing information relevance. Am J Health Behav. 2003;27(1):S227-S32.

44. Noar SM, Harrington NG, Aldrich RS. The role of message tailoring in the development of persuasive health communication messages. Ann Int Commun Assoc. 2009;33(1):73-133.

45. Bickmore TW, Caruso L, Clough-Gorr K, Heeren T. It's just like you talk to a friend'relational agents for older adults. Interact Comput. 2005;17(6):711-35.

46. Sillice MA, Morokoff PJ, Ferszt G, Bickmore T, Bock BC, Lantini R, et al. Using relational agents to promote exercise and sun protection: assessment of participants' experiences with two interventions. J Med Internet Res. 2018;20(2):e48.

47. Altman I,Taylor DA. Social penetration: the development of interpersonal relationships. New York: Holt, Rinehart \& Winston; 1973

48. Nass C, Steuer J, Tauber ER, editors. Computers are social actors. Proceedings of the SIGCHI conference on Human factors in computing systems; 1994.

49. Murdoch TB, Detsky AS. The inevitable application of big data to health care. Jama. 2013;309(13):1351-2.

\section{Publisher's Note}

Springer Nature remains neutral with regard to jurisdictional claims in published maps and institutional affiliations.

- fast, convenient online submission

- thorough peer review by experienced researchers in your field

- rapid publication on acceptance

- support for research data, including large and complex data types

- gold Open Access which fosters wider collaboration and increased citations

- maximum visibility for your research: over $100 \mathrm{M}$ website views per year

At BMC, research is always in progress.

Learn more biomedcentral.com/submissions 